\title{
ZUR HEIMATSBESTIMMUNG DES ANONYMUS SPERVOGEL.
}

Henrici stellt in seiner dissertation 'Zur geschichte der mbd. lyrik' s. 21 den satz auf, dass der dichter des älteren Spervogeltons ein Pfälzer sei. MSF. 27, $6 \mathrm{f}$. haben aber die handschriften den reim tuo: vruo, und da das metrum wie die construction hier sicher die zweisilbige adjectivform verlangen, bat Lachmann ohne zweifel richtig die therlieferung in tüeje : vrüeje gebessert. Die durch $j$ erweiterten formen des verbums tuon sind aber nur oberdeutsch, speciell alemannisch, vgl. Weinbold, Bair. gr. § 302, Alem. gr. § 354. - Der reim nieht : lieht MSF 28, 23 f. widerspricht der annahme alemannischer herkunft nicht, denn er kommt auch bei alemannischen dichtern vor, wenn er schon nach Paul, Mhd. gramm. § 113 besonders dem bairischen eigen ist.

Auch im wortschatz findet sich einiges beinahe ausschliesslich oberdeutsche. So ist egerde MSF 30,10 fast nur oberdeutsch, findet sich vereinzelt an der grenze des oberdeutschen und mitteldeutschen im bairischen Franken; nur ein beispiel ist im rein mitteldeutschen gebiet belegt (Grimm, wb. III, $347 \mathrm{f}$. Mhd. wb. I, 41 $1^{\text {b }}$ Lexer, Mbd. wb. I, 512, nachtr. 135, Schmeller, Bair. wb. I, 941, Staub und Tobler, Schweiz. idiot. I, 129).

sligele MSF 26, 19 kommt sonst, abgesehen von einer stelle im Passional, nur in oberdeutschen quellen vor, Mbd. wb. II, 2, 634 a, Lexer II, 1194.

künde MSF 30, 30 findet sich nur oberdeutsch, Mhd. wb. I, 312*, Lexer I, 1771, vgl. auch I, 1782.

Hiernach ist das aperçu Henrici's zurllekzuweisen und die heimat des anonymus im oberdeutschen sprachgebict, wahrscheinlich im alemannischen 7.0 suchen.

TÜBINGEN.

JOHN MEIER. 